\title{
BUBBLING OF ALMOST-HARMONIC MAPS BETWEEN 2-SPHERES AT POINTS OF ZERO ENERGY DENSITY *
}

\author{
Peter Topping
}

\begin{abstract}
We show that bubbling of almost-harmonic maps between 2-spheres has very different behaviour depending on whether or not bubbles develop at points in the domain at which the energy density of the body map is zero. We also see that this translates into different behaviour for the harmonic map flow. In [11] we obtained results, assuming nonzero bubble point density for certain bubbles, forcing the harmonic map flow to converge uniformly and exponentially to its limit. This involved proving a type of nondegeneracy for the harmonic map energy (a 'quantization' estimate') and an estimate on certain bubble scales (a 'repulsion' estimate). Here we show that without the nonzero bubble point density hypothesis, both the quantization and repulsion estimates fail, and we construct a flow in which the convergence is no longer exponentially fast.
\end{abstract}

\section{Introduction}

In this article, we study the harmonic map energy functional

$$
E(u):=\frac{1}{2} \int_{S^{2}}|\nabla u|^{2}
$$

for maps $u: S^{2} \rightarrow S^{2} \hookrightarrow \mathbb{R}^{3}$. We are interested in the behaviour of $E$ near its critical points known as harmonic maps - and near more general objects - known as bubble trees - which can be thought of as critical points at infinity. Such information is related to the behaviour of the $L^{2}$-gradient flow for $E$ - known as the harmonic map flow - which is a solution of

$$
\frac{\partial u}{\partial t}=\mathcal{T}(u(t))
$$

where the tension $\mathcal{T}(u) \in \Gamma\left(u^{*}\left(T S^{2}\right)\right)$ is defined to be the negation of the $L^{2}$-gradient of $E$. (See [11] or [4] for more details in this case.) The flow, when smooth, dissipates energy according to

$$
\frac{d}{d t} E(u(t))=-\|\mathcal{T}(u(t))\|_{L^{2}\left(S^{2}\right)}^{2},
$$

\footnotetext{
*This draft: October 2002.
} 
which integrates to the useful identity

$$
\int_{s}^{\infty}\|\mathcal{T}(u(t))\|_{L^{2}\left(S^{2}\right)}^{2} d t=E(u(s))-\lim _{t \rightarrow \infty} E(u(t))<\infty .
$$

Harmonic maps between 2-spheres are well-known to be precisely the rational maps and their complex conjugates (see $[4,(11.5)]$ ). In particular, being conformal maps from a surface, their energy is precisely the area of their image, and thus

$$
E(u)=4 \pi|\operatorname{deg}(u)| \in 4 \pi \mathbb{Z},
$$

for any harmonic $u$. Since the harmonic maps occur in families, the energy $E$ is degenerate near each critical point. However, by combining work of Gulliver-White [5] and L.Simon [7], [8, Lecture 3 , Section 2] it is possible to prove a substitute for nondegeneracy, that

$$
0 \leq E(u)-E(h) \leq C\|\mathcal{T}(u)\|_{L^{2}\left(S^{2}\right)}^{2},
$$

for $u$ sufficiently close ${ }^{1}$ to a harmonic map $h: S^{2} \rightarrow S^{2}$. Estimate (1.3) can be used to analyse the asymptotics of the harmonic map heat flow when it converges smoothly (without bubbling as described below) at infinite time.

In particular, for a flow converging smoothly to a limit $u_{\infty}$ as $t \rightarrow \infty$,

$$
\frac{\partial}{\partial t}\left[E(u(t))-E\left(u_{\infty}\right)\right]=-\|\mathcal{T}(u(t))\|_{L^{2}\left(S^{2}\right)}^{2} \leq-\frac{1}{C}\left[E(u(t))-E\left(u_{\infty}\right)\right]
$$

for sufficiently large $t$, and we see that $E(u(t))-E\left(u_{\infty}\right)$ must decay to zero exponentially fast. Moreover, (1.3) allows us to calculate

$$
-\frac{d}{d t}\left[E(u(t))-E\left(u_{\infty}\right)\right]^{\frac{1}{2}}=\frac{1}{2}\left[E(u(t))-E\left(u_{\infty}\right)\right]^{-\frac{1}{2}}\|\mathcal{T}(u(t))\|_{L^{2}\left(S^{2}\right)}^{2} \geq \frac{1}{2 \sqrt{C}}\|\mathcal{T}(u(t))\|_{L^{2}\left(S^{2}\right)},
$$

and thus, for $s \in[0, \infty)$ (and a new constant $C$ independent of $s$ )

$$
\int_{s}^{\infty}\|\mathcal{T}(u(t))\|_{L^{2}\left(S^{2}\right)} d t \leq C\left[E(u(s))-E\left(u_{\infty}\right)\right]^{\frac{1}{2}} .
$$

To begin with, this implies the finiteness of the left-hand side of (1.4) (which is simply false for the harmonic map flow in general, despite (1.2)). In addition, the exponential energy decay then forces the left-hand side of (1.4) to decay to zero exponentially fast. By returning to the equation (1.1) we then must have exponential convergence of $u(t)$ to $u_{\infty}$ as $t \rightarrow \infty$ in $L^{2}\left(S^{2}\right)$ and thus in $C^{k}$ - for any $k$ - by interpolation. (Even the convergence of $u(t)$ to $u_{\infty}$ in $L^{2}\left(S^{2}\right)$ uniformly as $t \rightarrow \infty$ is false in general for the harmonic map flow - see [10].)

Unfortunately, in general the harmonic map flow does not converge smoothly at infinite time (see [1] or the examples below). The best we can hope for is 'bubbling' convergence as the flow approaches a 'bubble tree' which can be thought of as a critical point at infinity for $E$. We first describe bubbling in the context of sequences of almost-harmonic maps. The following theorem is a combination of the seminal work of Struwe [9] and the improvements of Qing [6], Ding-Tian [3], and Wang [12]. See [11, Theorem 1.1] for a more sophisticated version.

\footnotetext{
${ }^{1}$ for example, close in $C^{3}$
} 
Theorem 1.1 Suppose that $u_{n}: S^{2} \rightarrow S^{2} \hookrightarrow \mathbb{R}^{3}$ is a sequence of smooth maps which satisfy $E\left(u_{n}\right)<M$ for some constant $M$, and all $n \in \mathbb{N}$, and $\mathcal{T}\left(u_{n}\right) \rightarrow 0$ in $L^{2}\left(S^{2}\right)$ as $n \rightarrow \infty$. Then we may pass to a subsequence in $n$, and find a harmonic map $u_{\infty}: S^{2} \rightarrow S^{2}$, and a set $\left\{x^{1}, \ldots, x^{m}\right\} \subset$ $S^{2}$ such that

(a) $u_{n} \rightarrow u_{\infty}$ weakly in $W^{1,2}\left(S^{2}\right)$,

(b) $u_{n} \rightarrow u_{\infty}$ strongly in $W_{l o c}^{2,2}\left(S^{2} \backslash\left\{x^{1}, \ldots, x^{m}\right\}\right)$.

Moreover, for each $x^{j}$, if we precompose each $u_{n}$ and $u_{\infty}$ with an inverse stereographic projection sending $0 \in \mathbb{R}^{2}$ to $x^{j} \in S^{2}$ (and continue to denote these compositions by $u_{n}$ and $u_{\infty}$ respectively) then for $i \in\{1, \ldots, k\}$ (for some $k \in \mathbb{N}$ dependent on $j$ ) there exist sequences $a_{n}^{i} \rightarrow 0 \in \mathbb{R}^{2}$ and $\lambda_{n}^{i} \downarrow 0$ as $n \rightarrow \infty$, and nonconstant harmonic maps $\omega^{i}: S^{2} \rightarrow S^{2}$ (which we precompose with the same inverse stereographic projection to view them also as maps $\mathbb{R}^{2} \cup\{\infty\} \rightarrow S^{2}$ ) such that:

(i)

$$
\frac{\lambda_{n}^{i}}{\lambda_{n}^{j}}+\frac{\lambda_{n}^{j}}{\lambda_{n}^{i}}+\frac{\left|a_{n}^{i}-a_{n}^{j}\right|^{2}}{\lambda_{n}^{i} \lambda_{n}^{j}} \rightarrow \infty
$$

as $n \rightarrow \infty$, for each unequal $i, j \in\{1, \ldots, k\}$.

(ii)

$$
\lim _{\mu \downarrow 0} \lim _{n \rightarrow \infty} E\left(u_{n}, D_{\mu}\right)=\sum_{i=1}^{k} E\left(\omega^{i}\right) .
$$

(iii) For each $i \in\{1, \ldots, k\}$ there exists a finite set of points $\mathcal{S} \subset \mathbb{R}^{2}$ (which may be empty, but could contain up to $k-1$ points) with the property that

$$
u_{n}\left(a_{n}^{i}+\lambda_{n}^{i} x\right) \rightarrow \omega^{i}(x),
$$

in $W_{l o c}^{2,2}\left(\mathbb{R}^{2} \backslash \mathcal{S}\right)$ as $n \rightarrow \infty$.

We refer to the map $u_{\infty}: S^{2} \rightarrow S^{2}$ as a 'body' map, and the maps $\omega^{i}: S^{2} \rightarrow S^{2}$ as 'bubble' maps, or simply 'bubbles.' The points $\left\{x^{1}, \ldots, x^{m}\right\}$ will be called 'bubble points,' and the $\lambda_{n}^{i}$ 'bubble scales.' Since each $\omega^{i}$ is a nonconstant harmonic map between 2 -spheres, the energy of each must be at least $4 \pi$.

In anticipation of an application of Theorem (1.1) to the harmonic map flow, we now seek a generalisation of (1.3); we want to understand the behaviour of $E$ for maps not necessarily $C^{k}$-close to a harmonic map, but with small tension in $L^{2}$. (Such maps need not be even $W^{1,2}$-close to a harmonic map - see [11].)

In [11, Theorem 1.2] we proved such a generalisation, building on our earlier work in [10]. Here we state a weaker version with simpler hypotheses. 
Theorem 1.2 Suppose that we have a sequence of maps $u_{n}: S^{2} \rightarrow S^{2}$ satisfying the hypotheses of Theorem 1.1, and that we pass to a subsequence and find a limit $u_{\infty}$, bubble points $\left\{x^{j}\right\}$ and bubble data $\omega^{i}, \lambda_{n}^{i}, a_{n}^{i}$ at each bubble point - as we know we can from Theorem 1.1.

Suppose that at each bubble point, only one bubble develops - i.e. that in the language of Theorem $1.1, k=1$ at each $x^{j}$. Suppose further that the energy density of the body map is nonzero at each bubble point - i.e. that $\left|\nabla u_{\infty}\right|\left(x^{j}\right) \neq 0$ for all $j \in\{1, \ldots, m\}$.

Then there exist constants $C>0$ and $k \in \mathbb{N} \cup\{0\}$ such that after passing to a subsequence, the energy is quantized according to

$$
\left|E\left(u_{n}\right)-4 \pi k\right| \leq C\left\|\mathcal{T}\left(u_{n}\right)\right\|_{L^{2}\left(S^{2}\right)}^{2} .
$$

A further part of [11, Theorem 1.2] is a 'repulsion' estimate which controls some of the bubble scales $\lambda_{n}^{i}$ by:

$$
\lambda_{n}^{i} \leq \exp \left[-\frac{1}{C\left\|\mathcal{T}\left(u_{n}\right)\right\|_{L^{2}\left(S^{2}\right)}^{2}}\right] .
$$

Remark 1.3 The full version of [11, Theorem 1.2] allows multiple bubbling at each point subject to a compatibility condition, and only requires the body map to have zero energy density at certain bubble points. It is at these bubble points that the bubble scales satisfy the repulsion estimate (1.6).

One of the results of this paper is that both the quantization estimate (1.5) and the repulsion estimate (1.6) may fail if we allow arbitrary bubbles to occur at points where the energy density of $u_{\infty}$ is zero. See Corollary 2.2 below.

Theorem 1.1 gives us a basic understanding of the asymptotics of the harmonic map flow, as we now describe. By considering (1.2), given any sufficiently regular harmonic map flow $u$, we may find a sequence of times $t_{n} \rightarrow \infty$ such that $\mathcal{T}\left(u\left(t_{n}\right)\right) \rightarrow 0$ in $L^{2}\left(S^{2}\right)$. We may then apply Theorem 1.1 to the sequence of maps $\left\{u\left(t_{n}\right)\right\}$ to get bubbling convergence. (So far, this says nothing about the behaviour of the flow at times between $t_{n}$ and $t_{n+1}$.)

It turns out to be possible (see [11] for details) to apply Theorem 1.2 to flows with appropriate bubbling, in order to obtain a quantization estimate for $u(t)$ for all sufficiently large $t$ - not just for the maps $u\left(t_{n}\right)$. We state this result for smooth flows - note that the standard Struwe flow (as in [9]) is smooth for sufficiently large time, and we are only concerned here with the asymptotics of the flow. As before, the results in [11] are somewhat stronger.

Theorem 1.4 Suppose that $u: S^{2} \times[0, \infty) \rightarrow S^{2}$ is a smooth solution of (1.1). Then after picking times $t_{n} \rightarrow \infty$ so that $\mathcal{T}\left(u\left(t_{n}\right)\right) \rightarrow 0$ in $L^{2}\left(S^{2}\right)$ and analysing the sequence $\left\{u\left(t_{n}\right)\right\}$ with Theorem 
1.1, if only one bubble develops at each bubble point and the energy density of the body map $u_{\infty}$ is nonzero at each bubble point, then there exists $C>0$ independent of $t$ such that

$$
0 \leq E(u(t))-\bar{E} \leq C\|\mathcal{T}(u(t))\|_{L^{2}\left(S^{2}\right)}^{2},
$$

for sufficiently large $t$, where

$$
\bar{E}:=\lim _{t \rightarrow \infty} E(u(t)) \in 4 \pi \mathbb{Z}
$$

Remark 1.5 Exactly as described earlier for smoothly converging flows, we can use an estimate such as (1.7) to deduce exponential decay of both $[E(u(t))-\bar{E}]$ and $\int_{t}^{\infty}\|\mathcal{T}(u(s))\|_{L^{2}\left(S^{2}\right)} d s$. This time, the consequence is the following corollary (cf. [11, Theorem 1.7]).

Corollary 1.6 Under the hypotheses of Theorem 1.4, for any $k \in \mathbb{N}$ and $\Omega \subset \subset S^{2} \backslash\left\{x^{1}, \ldots, x^{m}\right\}$ (where $\left\{x^{1}, \ldots, x^{m}\right\}$ are the bubble points) we have exponential decay of

$$
\left\|u(t)-u_{\infty}\right\|_{L^{2}\left(S^{2}\right)}+\left\|u(t)-u_{\infty}\right\|_{C^{k}(\Omega)}
$$

as $t \rightarrow \infty$.

A priori, not even uniform convergence $u(t) \rightarrow u_{\infty}$ in $L^{2}\left(S^{2}\right)$ as $t \rightarrow \infty$ is clear. In fact, for more elaborate target manifolds than $S^{2}$, this may fail - see [10].

A further result of this paper is that if we allow a smooth flow to develop bubbles at points in the domain where the energy density of $u_{\infty}$ is zero, then the convergence of the flow need not be exponential - see Theorem 2.3 below. The combination of this result and [11] leads to apparently the first situation for the harmonic map flow in which the position of bubble points in the domain is shown to have a bearing on the properties of the bubbling.

\section{Details of results}

We first need the results of some energy and tension computations for a simple map between 2-spheres.

Proposition 2.1 Given $d \in \mathbb{N}$ and $\varepsilon \in(0,1]$, consider the map $w_{\varepsilon}: S^{2} \rightarrow S^{2}$ defined in terms of stereographic complex coordinates by

$$
w_{\varepsilon}(z)=\varepsilon^{d}\left(z^{d}-\bar{z}^{-d}\right) .
$$

Then 
(a)

$$
c_{0} d \varepsilon^{2 d} \leq E\left(w_{\varepsilon}\right)-8 \pi d \leq C_{0} d \varepsilon^{2 d},
$$

for universal $c_{0}, C_{0}>0$;

(b)

$$
c_{1} \varepsilon^{2 d-1} \leq\left\|\mathcal{T}\left(w_{\varepsilon}\right)\right\|_{L^{2}\left(S^{2}\right)} \leq C_{1} \varepsilon^{2 d-1},
$$

for $c_{1}, C_{1}>0$ dependent only on $d$.

Given these calculations (see Section 3 for comments on the details) we may define a sequence of maps $u_{n}: S^{2} \rightarrow S^{2}$ by $u_{n}:=w_{1 / n}$ to establish the following corollary.

Corollary 2.2 For any $d \in \mathbb{N}$, there exist $k \in \mathbb{N}$ and a sequence of smooth maps $u_{n}: S^{2} \rightarrow S^{2}$ with $\mathcal{T}\left(u_{n}\right) \rightarrow 0$ in $L^{2}\left(S^{2}\right)$, such that

$$
c\left\|\mathcal{T}\left(u_{n}\right)\right\|_{L^{2}\left(S^{2}\right)}^{\frac{2 d}{2 d-1}} \leq\left|E\left(u_{n}\right)-4 \pi k\right| \leq C\left\|\mathcal{T}\left(u_{n}\right)\right\|_{L^{2}\left(S^{2}\right)}^{\frac{2 d}{2 d-1}},
$$

with $c, C>0$ dependent only on $d$. In particular, the quantization estimate (1.5) fails for the sequence $\left\{u_{n}\right\}$. Moreover, if we apply Theorem 1.1 to the sequence $\left\{u_{n}\right\}$, then the resulting bubble scales must satisfy

$$
c\left\|\mathcal{T}\left(u_{n}\right)\right\|_{L^{2}\left(S^{2}\right)}^{\frac{1}{2 d-1}} \leq \lambda_{n} \leq C\left\|\mathcal{T}\left(u_{n}\right)\right\|_{L^{2}\left(S^{2}\right)}^{\frac{1}{2 d-1}},
$$

for some $c, C>0$ independent of $n$.

The second part of this corollary should be compared to the repulsion estimate in [11] mentioned in (1.6).

The sequence $u_{n}$ converges to a constant body map with bubbles of degree $d$ and $-d$. An alternative example, with slightly different exponents, would arise by defining instead $u_{n}(z)=w_{1 / n}(n z)$. In this case we would get a degree $d$ body map with a single degree $-d$ bubble. Next we state precisely our result for the harmonic map flow.

Theorem 2.3 Fix an integer $d>1$ and define $u_{0}: S^{2} \rightarrow S^{2}$ by

$$
u_{0}(z)=z^{d}-\bar{z}^{-d} .
$$

Then there exist $\eta=\eta(d)>0$ and a unique smooth solution $u: S^{2} \times[0, \infty) \rightarrow S^{2}$ of the harmonic map heat equation (1.1) with $u(0)=u_{0}$, and for $t \geq 1$ the solution has the following properties:

(a)

$$
\left\|u(t)-u_{\infty}\right\|_{L^{2}\left(S^{2}\right)} \geq \eta t^{-\frac{1}{2(d-1)}}
$$


(b)

$$
\|\mathcal{T}(u(t))\|_{L^{2}\left(S^{2}\right)} \geq \eta t^{-\frac{d}{d-1}}
$$

(c)

$$
\int_{t}^{\infty}\|\mathcal{T}(u(s))\|_{L^{2}\left(S^{2}\right)} d s \geq \eta t^{-\frac{1}{d-1}}
$$

(d)

$$
E(u(t))-\lim _{s \rightarrow \infty} E(u(s)) \geq \eta t^{-\frac{d+1}{d-1}}
$$

Inequality (2.3) implies that $u(t)$ does not converge exponentially to $u_{\infty}$, and should be compared to $(1.8)$. (Note that for the flow in question, $u(t)$ does nevertheless converge to $u_{\infty}$ as $t \rightarrow \infty$.)

Inequalities (2.5) and (2.6) imply in particular that their left-hand sides cannot decay exponentially to zero as $t \rightarrow \infty$, and should be compared to Remark 1.5.

\section{Elements of the calculations and proofs}

Although it is the complex coordinates which help us to pick natural maps for this problem, we are better off calculating in spherical polar coordinates $(\theta, \phi)$ in order to later apply the comparison principle and exploit rotational symmetry. With our convention, $\theta \in[0, \pi]$ represents the 'latitudinal' angle from the vertical, and $\phi$ measures longitude.

We will be considering, for integral $d \geq 1$, maps of the form

$$
(\theta, \phi) \rightarrow(\beta(\theta), d \phi)
$$

for appropriate $\beta:[0, \pi] \rightarrow \mathbb{R}$. The energy of such a map is given by

$$
E=\pi \int_{0}^{\pi}\left[\left(\beta^{\prime}(\theta)\right)^{2} \sin \theta+\frac{d^{2}}{\sin \theta} \sin ^{2} \beta(\theta)\right] d \theta,
$$

and the tension may be written

$$
\mathcal{T}=\tau(\beta) \frac{\partial}{\partial \beta}
$$

where $\tau(\beta):[0, \pi] \rightarrow \mathbb{R}$ may be calculated ${ }^{2}$ to be

$$
\tau(\beta)=\beta^{\prime \prime}(\theta)+\frac{1}{\tan \theta} \beta^{\prime}(\theta)-\frac{d^{2} \sin \beta(\theta) \cos \beta(\theta)}{\sin ^{2} \theta},
$$

and we have abused notation by using $\beta$ both as a function and as a target coordinate.

\footnotetext{
${ }^{2}$ cf. the case $d=1$ in, say, [1]
} 
Proof. (Proposition 2.1.) The map $w_{\varepsilon}$ of Proposition 2.1 is of the form (3.1) with $\beta \equiv \alpha$ where

$$
\alpha(\theta)=\alpha_{\varepsilon}(\theta):=2 \tan ^{-1}\left[\varepsilon^{d}\left(\tan ^{d} \frac{\theta}{2}-\tan ^{-d} \frac{\theta}{2}\right)\right] .
$$

Plugging this into (3.2) and using the substitution $t=\tan \frac{\theta}{2}$, we eventually find that

$$
E-8 \pi d=32 \pi d^{2} \varepsilon^{2 d} \int_{0}^{1} \frac{t^{2 d-1}}{\left(1+\varepsilon^{2 d}\left(t^{d}-t^{-d}\right)^{2}\right)^{2}} d t,
$$

which then gives $(2.1)$ of the proposition since the integral can easily be checked to be of order $\frac{1}{d}$ uniformly for $\varepsilon \in(0,1]$.

From the definitions of $\alpha$ and $\tau$, a straightforward but long calculation takes us to

$$
\tau(\alpha)(\theta)=\frac{-4 \varepsilon^{3 d} d^{2} \sec ^{4} \frac{\theta}{2}\left(\tan ^{d-2} \frac{\theta}{2}-\tan ^{-d-2} \frac{\theta}{2}\right)}{\left(1+\varepsilon^{2 d}\left(\tan ^{d} \frac{\theta}{2}-\tan ^{-d} \frac{\theta}{2}\right)^{2}\right)^{2}} .
$$

Plugging this into the expression

$$
\left\|\mathcal{T}\left(w_{\varepsilon}\right)\right\|_{L^{2}\left(S^{2}\right)}^{2}=2 \pi \int_{0}^{\pi}|\tau|^{2} \sin \theta d \theta
$$

leads us to (2.2) of the proposition, with the integral being dominated now by values of $\theta$ of the order of $\varepsilon$.

Remark 3.1 Related to $w_{\varepsilon}$ is the map $u_{\varepsilon}: S^{2} \rightarrow S^{2}$ given by

$$
u_{\varepsilon}(z)=\varepsilon^{d}\left(z^{d}+\bar{z}^{-d}\right) .
$$

Calculations for $u_{\varepsilon}$ show (cf. Proposition 2.1) that

$$
-c_{0} d \varepsilon^{2 d} \leq E\left(u_{\varepsilon}\right)-8 \pi d \leq-C_{0} d \varepsilon^{2 d}
$$

for universal $c_{0}, C_{0}>0$, and

$$
c_{1} \varepsilon^{2 d-1} \leq\left\|\mathcal{T}\left(u_{\varepsilon}\right)\right\|_{L^{2}\left(S^{2}\right)} \leq C_{1} \varepsilon^{2 d-1}
$$

for $c_{1}, C_{1}>0$ dependent only on $d$. This turns out to be useful in the theory of biharmonic maps - see $[2]$.

We now consider the significance of the maps $w_{\varepsilon}$ to the harmonic map heat flow. As we see in the following proposition, it is possible to use these maps, with $\varepsilon$ dependent on time, as barriers.

Proposition 3.2 Let $\varepsilon:[0, \infty) \rightarrow(0,1]$ solve the $O D E \dot{\varepsilon}=-16 d \varepsilon^{2 d-1}$ with initial condition $\varepsilon(0)=1$. Then

$$
\frac{\partial}{\partial t} \alpha_{\varepsilon(t)}(\theta)-\tau\left(\alpha_{\varepsilon(t)}\right)(\theta) \geq 0
$$

for $\theta \in\left[0, \frac{\pi}{2}\right], t \geq 0$ 
By symmetry, the left-hand side of (3.9) must be weakly negative for $\theta \in\left[\frac{\pi}{2}, \pi\right]$. The proof of the proposition is a straightforward calculation using the inequality $r^{2}\left(1+\varepsilon^{2 d}\left(r^{d}-r^{-d}\right)^{2}\right) \geq \varepsilon^{2} / 2$ valid for $r>0, \varepsilon \in(0,1], d \geq 1$, say. Note that neither this inequality nor the ODE of the proposition is sharp.

Remark 3.3 If we now have a smooth solution of (1.1) of the form

$$
(\theta, \phi, t) \rightarrow(h(\theta, t), d \phi)
$$

where $h:[0, \pi] \times[0, T] \rightarrow \mathbb{R}$ satisfies $h(0, \cdot) \equiv-\pi, h(\theta, \cdot) \equiv-h(\pi-\theta, \cdot)$ and $h(\theta, 0) \leq \alpha_{1}(\theta)$ for $\theta \in\left[0, \frac{\pi}{2}\right]$ then by virtue of Proposition 3.2, we may apply the comparison principle (see [1] for details in a very similar situation) to establish that

$$
h(\theta, t) \leq \alpha_{\varepsilon(t)}(\theta)
$$

for $\theta \in\left[0, \frac{\pi}{2}\right]$ and $t \in[0, T]$, with an analogous (reversed) inequality for $\theta \in\left[\frac{\pi}{2}, \pi\right]$. Similarly, if $h(\theta, 0) \in[-\pi, \pi]$ for $\theta \in[0, \pi]$, then the comparison principle tells us that $h(\theta, t) \in[-\pi, \pi]$ for $\theta \in[0, \pi]$ and $t \in[0, T]$.

Proof. (Theorem 2.3.) The work of Struwe [9] gives the existence of a weak solution $u$ with $u(0)=u_{0}$, which is smooth on some maximal time interval $[0, T)$ with $T \in(0, \infty]$, and indeed smooth throughout space-time except possibly at finitely many points. There is no other smooth solution on $[0, T)$ with $u_{0}$ as initial map - see for example [9].

Since the initial map is of the form (3.1) with $\beta \equiv \alpha_{1}$, and $\alpha_{1}(\theta)=-\alpha_{1}(\pi-\theta)$, the uniqueness we have described guarantees that the flow will be of the form (3.10) for $t \in[0, T)$, with $h$ satisfying $h(0, \cdot) \equiv-\pi$ and $h(\theta, \cdot) \equiv-h(\pi-\theta, \cdot)$ for $\theta \in\left[0, \frac{\pi}{2}\right]$. By the finiteness of the singular set, a flow of this form must be smooth away from the poles $\theta=0$ and $\theta=\pi$ at time $t=T$ if $T<\infty$, and so by the bubbling description of singularities in the Struwe flow given in [9], the only way that $u$ could fail to be smooth would be by bubbling at the poles.

Meanwhile, since $h(\cdot, 0) \equiv \alpha_{1}$, Remark 3.3 tells us that $-\pi \leq h(\theta, t) \leq \alpha_{\varepsilon(t)}(\theta)$ for $\theta \in\left[0, \frac{\pi}{2}\right]$ and $t \in[0, T)$, where $\varepsilon(t)>0$ is as in Proposition 3.2. In particular, this restricts the image of the flow near the pole $\theta=0$ at finite time, ruling out the development of a bubble at finite time and forcing $T=\infty$. Note that our knowledge of the harmonic maps between 2 -spheres, and the bubbling theory of Struwe then forces the flow limit $u_{\infty}$ to be the constant map $(\theta, \phi) \rightarrow(0, d \phi)$.

Although this argument holds for all integral $d \geq 1$, we are assuming $d>1$ in the theorem, and therefore the solution of the ODE for $\varepsilon$ in Proposition 3.2 is

$$
\varepsilon(t)=(1+32 d(d-1) t)^{-\frac{1}{2(d-1)}} .
$$

For $t \geq 1$, say, we have

$$
\varepsilon(t) \geq \eta t^{-\frac{1}{2(d-1)}},
$$

for some $\eta=\eta(d)>0$ which we may reduce during the proof. 
Meanwhile, for $0 \leq \theta \leq \varepsilon \leq 1$, we may estimate

$$
\alpha_{\varepsilon}(\theta) \leq-\eta
$$

for some small $\eta>0$ independent of $\varepsilon$, and therefore, since $\alpha_{\varepsilon(t)}(\theta) \geq h(\theta, t)$ for this range of $\theta$, and all $t \geq 0$, we have $\left|u(t)-u_{\infty}\right| \geq \eta>0$ (for a possibly smaller $\eta$ ) on the disc defined by $\theta \leq \varepsilon(t)$. Consequently,

$$
\left\|u(t)-u_{\infty}\right\|_{L^{2}\left(S^{2}\right)} \geq \eta \varepsilon(t),
$$

which once combined with (3.11) is (2.3) of Theorem 2.3.

In order to tackle (2.4) let us return to the definition of $\tau$ given in (3.3), and assume that $\beta(0)=-\pi$ and $\beta\left(\frac{\pi}{2}\right)=0$. Multiplying this definition by $\sin ^{2} \theta \beta^{\prime}(\theta)$ and integrating from $\theta=0$ to $\theta=\frac{\pi}{2}$, we obtain (after integrating by parts)

$$
\int_{0}^{\frac{\pi}{2}} \sin ^{2} \theta \tau \beta^{\prime}(\theta) d \theta=\frac{1}{2}\left(\beta^{\prime}\left(\frac{\pi}{2}\right)\right)^{2} .
$$

Recalling (3.2) and (3.6), Cauchy-Schwarz then tells us that

$$
\left(\beta^{\prime}\left(\frac{\pi}{2}\right)\right)^{2} \leq 2 \int_{0}^{\frac{\pi}{2}} \sin \theta \tau \beta^{\prime}(\theta) d \theta \leq C E^{\frac{1}{2}}\|\mathcal{T}\|_{L^{2}}
$$

When we apply this to flow $u$ and its corresponding $h$, keeping in mind the relationship of $h(\cdot, t)$ and $\alpha_{\varepsilon(t)}$, we find that

$$
\|\mathcal{T}(u(t))\|_{L^{2}\left(S^{2}\right)} \geq \eta\left(\frac{\partial h}{\partial \theta}\left(\frac{\pi}{2}, t\right)\right)^{2} \geq \eta\left(\alpha_{\varepsilon(t)}^{\prime}\left(\frac{\pi}{2}\right)\right)^{2} \geq \eta \varepsilon^{2 d},
$$

where the value of $\eta=\eta(d)>0$ is allowed to decrease at each instance. This establishes (2.4).

The final two parts of Theorem 2.3 follow easily from (2.4). Straightforward integration gives (2.5), whilst integrating the square of (2.4), keeping in mind (1.2), gives (2.6).

Remark 3.4 Only in the case $d>1$ does the solution $\varepsilon(t)$ of the ODE in Proposition 3.2 fail to decay exponentially as required for Theorem 2.3. However, $\alpha_{\varepsilon(t)}$ serves as a natural barrier even for $d=1$, preventing finite time blow-up - cf. Chang-Ding [1].

\section{References}

[1] K.-C. CHANG and W.-Y. DING, A result on the global existence for heat flows of harmonic maps from $D^{2}$ into $S^{2}$. 'Nematics.' J.-M. Coron et al ed., Kluwer Academic Publishers (1990) $37-48$.

[2] N. COURSE, Thesis due 2003/2004, University of Warwick. 
[3] W.-Y. DING and G. TIAN, Energy identity for a class of approximate harmonic maps from surfaces. Comm. Anal. Geom. 3 (1995) 543-554.

[4] J. EELLS and L. LEMAIRE, A report on harmonic maps. Bull. London Math. Soc. 10 (1978) $1-68$.

[5] R. GULLIVER and B. WHITE, The Rate of Convergence of a Harmonic Map at a Singular Point. Math. Ann. 283 (1989) 539-549.

[6] J. QING, On singularities of the heat flow for harmonic maps from surfaces into spheres. Comm. Anal. Geom. 3 (1995) 297-315.

[7] L. SIMON, Asymptotics for a class of nonlinear evolution equations, with applications to geometric problems. Ann. Math. 118 (1983) 525-571.

[8] L. SIMON, Theorems on the Regularity and Singularity of Minimal Surfaces and Harmonic Maps. 'Geometry and Global Analysis.' T. Kotake et al ed., MSJ report, Sendai, Japan (1993) 111-145.

[9] M. STRUWE, On the evolution of harmonic mappings of Riemannian surfaces. Comment. Math. Helv. 60 (1985) 558-581.

[10] P. M. TOPPING, Rigidity in the Harmonic Map Heat Flow. J. Differential Geometry 45 (1997) 593-610.

[11] P. M. TOPPING, Repulsion and quantization in almost-harmonic maps, and asymptotics of the harmonic map flow. To appear, Annals of Math.

[12] C.-Y. WANG, Bubble phenomena of certain Palais-Smale sequences from surfaces to general targets. Houston J. Math. 22 (1996) 559-590.

topping@maths . warwick.ac.uk

http://www.maths. warwick.ac.uk/ ${ }^{\sim}$ topping

Mathematics Institute, University of Warwick, Coventry, CV4 7AL, U.K. 